\title{
Comparative Effectiveness of Trimethoprim- Sulfamethoxazole Versus Atovaquone for the Prophylaxis of Pneumocystis Pneumonia in Patients with Connective Tissue Diseases Receiving Prolonged High-Dose Glucocorticoids
}

Sadao Jinno ( $\square$ sadaoj@gmail.com )

Kobe Daigaku Igakubu Fuzoku Byoin https://orcid.org/0000-0003-3021-183X

\section{Kengo Akashi}

Kobe University Graduate School of Medicine School of Medicine: Kobe Daigaku Daigakuin Igakukei Kenkyuka Igakubu

\section{Akira Onishi}

Kobe University Graduate School of Medicine School of Medicine: Kobe Daigaku Daigakuin Igakukei Kenkyuka Igakubu

\section{Yoko Nose}

Kobe University Graduate School of Medicine School of Medicine: Kobe Daigaku Daigakuin Igakukei Kenkyuka Igakubu

\section{Mai Yamashita}

Kobe University Graduate School of Medicine School of Medicine: Kobe Daigaku Daigakuin Igakukei Kenkyuka Igakubu

\section{Jun Saegusa}

Kobe University Graduate School of Medicine School of Medicine: Kobe Daigaku Daigakuin Igakukei Kenkyuka Igakubu

\section{Research article}

Keywords: pneumocystis pneumonia, prophylaxis, trimethoprim-sulfamethoxazole, atovaquone, connective tissue diseases

Posted Date: June 2nd, 2021

DOI: https://doi.org/10.21203/rs.3.rs-576984/v1

License: (c) (i) This work is licensed under a Creative Commons Attribution 4.0 International License. Read Full License 


\section{Abstract}

Background: We compared the prophylactic effect of trimethoprim-sulfamethoxazole (TMP-SMX) with atovaquone for pneumocystis pneumonia (PCP) in patients with connective tissue diseases (CTDs) receiving high-dose glucocorticoids.

Methods: Patients with CTDs aged $\geq 18$ years who were treated with a prolonged course ( $\geq 4$ weeks) of glucocorticoids ( $\geq 20 \mathrm{mg} /$ day prednisone) in a Japanese tertiary center between 2013 and 2017 were included. The patients were categorized into two groups: TMP-SMX and atovaquone group. Adjusted cumulative incidence of PCP was compared between the two groups after propensity score weighting for differences in confounding factors.

Results: A total of 480 patients with a prolonged high-dose glucocorticoid treatment were identified. Out of 383 patients with TMP-SMX prophylaxis, 102 (26.8\%) patients experienced adverse events leading to discontinuation within 4 weeks of initiation while no patient in the atovaquone discontinued the therapy. Two hundred eighty-one patients received TMP-SMX while 107 received atovaquone for PCP prophylaxis. During a total of 397.0 person-years, 7 PCP cases ( 2 in the TMP-SMX, 5 in the atovaquone) occurred with a mortality rate of $54.5 \%$. After adjusting for differences in baseline characteristics, the adjusted cumulative incidence of PCP was similar between the two group $(\mathrm{HR}=0.97,95 \% \mathrm{Cl}=0.19$ to $5.09, \mathrm{p}=$ 0.97).

Conclusion: Prophylactic effects for PCP in CTDs patients receiving prolonged high-dose glucocorticoids were similar between TMP-SMX and atovaquone. Atovaquone was well-tolerated with no side effects.

\section{Background}

Pneumocystis pneumonia (PCP) is a potentially life-threatening opportunistic infection in patients with connective tissue diseases (CTDs) who are on immunosuppressive agents. PCP prophylaxis is generally recommended for immune compromised patients without HIV infection receiving a glucocorticoid (GC) dose equivalent to $\geq 20 \mathrm{mg}$ of prednisone daily for 4 weeks or longer [1]. Trimethoprim-sulfamethoxazole (TMP-SMX) is regarded as a preferred agent for PCP prophylaxis. The prophylactic efficacy of TMP-SMX on PCP among CTDs patients has been reported [2,3]. Although TMP-SMX is considered to be welltolerated among non-HIV patients in whom adverse effects range between 6 to $8 \%[4,5]$, treatment cessation is required for some patients due to severe adverse events (AEs) such as neutropenia, anaphylaxis, or skin rash. Alternatively, other agents such as atovaquone or aerosolized pentamidine can be used for PCP prophylaxis, but data regarding its efficacy among CTDs patients have been scarce [6, 7]. No firm conclusions can be reached about the efficacy of other PCP prophylaxis agents since these studies involved small number of CTDs patients and only a few developed PCP.

Given potentially serious AEs of TMP-SMX leading to discontinuation on certain patient population, it is important to know if other PCP prophylactic agents can be used effectively and safely in patients with 
CTDs. The objective of this study was to compare the prophylactic effect of TMP-SMX with atovaquone in patients with CTDs receiving high-dose GCs.

\section{Materials And Methods}

\section{Patients and data collection}

The electronic medical database at Kobe University Hospital was examined, and patients with CTDs aged $\geq 18$ years who were treated with a prolonged course ( $\geq 4$ weeks) of GC ( $\geq 20 \mathrm{mg} /$ day prednisolone) between 2013 and 2017 were identified. Patients with a history of PCP, HIV infection, current cancer, or a solid organ transplant were excluded. The baseline date was defined as the first day of initiating high-dose GC. The observation period for each treatment episode was 1 year from the baseline date because previous studies suggested that most PCP cases occur within one-year period [8]. Patient consent was waived by the institutional review board of Kobe University Hospital due to the retrospective nature of the study (approval number: B190296).

The patients were categorized into two groups: TMP-SMX and atovaquone. If patients had to change or stop the 1 st -line prophylactic drug because of AEs within 4 weeks after 1 st -line regimen started, they were assigned to 2 nd -line regimen group.

\section{Medication, Dosage, And Duration Of Pcp Prophylaxis}

As prophylaxis for PCP, 1 single-strength tablet of TMP-SMX was administrated daily, which contained $80 \mathrm{mg}$ of TMP and $400 \mathrm{mg}$ of SMX. The dose reduction was allowed if patients experienced AEs. Patients who could not take TMP-SMX were given oral atovaquone suspension daily at a dose of $1500 \mathrm{mg}$ or aerosolized pentamidine at a dose of $300 \mathrm{mg}$ every 4 weeks. Dapsone was not permitted for use as PCP prophylaxis in Japan during the study period. Prophylaxis was discontinued according to attending physicians' decision when GC dose was reduced below $20 \mathrm{mg}$ of prednisolone equivalent dose.

\section{The Definition Of Pcp}

The diagnosis of PCP required recent history of dyspnea, exertion of nonproductive cough, appropriate response to PCP treatment, the absence of evidence of other cause, and radiographic findings compatible with PCP. The diagnosis was considered definitive if Pneumocystis.jirovecii was found on microscopical analysis of sputum or bronchoalveolar lavage fluid. The diagnosis was considered presumptive if serum $\beta$-D-glucan was elevated when microscopical analysis were not available [9]. Both definitive and presumptive cases were included in this study.

\section{Statistical analysis}


For baseline characteristics, categorical values were evaluated with Mantel-Haenszel Chi-Square test. Continuous values were evaluated with ANOVA. The primary outcome was incidence of PCP during the observation. A propensity score approach was used to account for differences between the TMP-SMX and the atovaquone in potential confounding factors and predictors of outcome. The logistic regression model was used to calculate the propensity score defined as the probability of a patient receiving TMPSMX or atovaquone based on patient covariates. Prespecified covariates were baseline age, sex, diagnosis (systemic lupus erythematosus, idiopathic inflammatory myopathy, ANCA-associated vasculitis and other CTDs), disease duration, initial PSL dose, lymphocytopenia defined as below 800 cells /ml [10], diabetes mellitus, chronic lung disease, and use of sulfasalazine, steroid pulse therapy, cyclophosphamide, biologics, and other immunosuppressive agents such as azathioprine, methotrexate, mizoribine or mycophenolate. The analyses were based on inverse probability of treatment weighting to reduce the variability of weights and standard errors of estimated treatment effects. Because deaths irrespective to PCP was considered as a competing risk of PCP incidence, we used the Fine-Gray hazard competing risk regression model for the primary outcome. To account for missing data, we used multiple imputations by a chained equation, using all other variables to impute any missing values for variables included in the logistic regression model. We generated 5 independent imputed datasets. For each dataset, we estimated propensity score from the logistic model and pooled the resulting parameters according to Rubin's rules. Statistical analyses were performed using STATA version 13.1 (StataCorp, Texas, USA) and R version 4.0.1 (R Development Core Team, Vienna, Austria) and $p<0.05$ was considered statistically significant.

\section{Results}

A total of 480 patients with CTDs with a prolonged high-dose GC treatment were identified. Fifteen patients were excluded since they were already taking TMP-SMX at the time of identification. As a $1 \mathrm{st}$ line agent, 383 patients initiated TMP-SMX while 17 did other prophylactic drugs ( 12 atovaquone and 5 pentamidine). Sixty-five patients underwent a high-dose GC treatment without PCP prophylaxis. Out of 383 patients with TMP-SMX prophylaxis, 102 (26.6\%) patients experienced AEs leading to discontinuation within 4 weeks of initiation. Major AEs were cytopenia in 44 cases (11.5\%), skin rash in 24 cases $(6.3 \%)$, liver function test abnormality in 23 cases $(6.0 \%)$, renal function test abnormality in 16 cases (4.2\%), and electrolyte abnormality in 11 cases (2.9\%). Out of 102 patients who discontinued TMPSMX, 98 switched to other prophylactic drugs ( 95 atovaquone and 3 pentamidine), and 4 stopped PCP prevention. On the other hands, no patients in the atovaquone discontinued prophylaxis due to AEs during the study period. Subsequently, patients were categorized as follows; 281 patients to TMP-SMX group, and 107 patients to atovaquone group (Fig. 1). Among 281 patients in the TMP-SMX, 142 patients tolerated TMP-SMX, one oral single-strength tablet per day. However, the rest of patients needed to reduce the dose because of AEs.

Baseline characteristics when GC treatment was started, were shown in Table 1. Compared with the TMPSMX, patients in the atovaquone were older, more likely to have chronic lung disease and lymphocytopenia, to be treated with steroid pulse therapy, and cyclophosphamide. Death except for PCP 
occurred more commonly in the atovaquone. To avoid treatment-selection bias, inverse probability of treatment weighting was performed. In the weighted cohort, these baseline characteristics of the two groups were statistically comparable (Supplementary table S1).

During a total of 397.0 person-years (307.7 persons-years in the TMP-SMX and 89.3 persons-years in the atovaquone), 7 PCP cases ( 2 in the TMP-SMX and 5 in the atovaquone) occurred with a mortality rate of $54.5 \%$. There were 31 deaths irrelevant to PCP (17 in the TMP-SMX and 14 in the atovaquone). After adjusting for differences in baseline characteristics between the two groups, the adjusted cumulative incidence of PCP was similar between the two group ( $\mathrm{HR}=0.97,95 \% \mathrm{Cl}=0.19$ to $5.09, \mathrm{p}=0.97)$ (Fig. 2).

\section{Discussion}

In this single-center analysis, prophylactic effects for PCP in CTDs patients receiving prolonged high-dose GCs were similar between TMP-SMX and atovaquone. To the best of our knowledge, this is the first article to compare the effects of atovaquone with TMP-SMX in CTDs patients.

Previously, a few studies compared the prophylactic effects of TMP-SMX with atovaquone in patients without CTD $[11,12]$. One prospective trial compared TMP-SMX to atovaquone for PCP prophylaxis in 39 patients (double-strength TMP-SMX daily [ $n=19]$ or $1500 \mathrm{mg}$ of atovaquone daily $[n=20]$ ) following autologous hematopoietic cell transplantation [12]. There were no cases of PCP in either group at 12 months post-transplant. Another trial involving 185 renal transplant recipients (single-strength TMP-SMX daily [ $n=160$ ] or $1500 \mathrm{mg}$ of atovaquone daily [ $=25]$ ) also found no cases of PCP in either group at 12 months post-transplant [11]. The need for dose reduction and/or premature discontinuation of therapy secondary to AEs was more common in TMP-SMX-treated patients in both studies. Among HIV-infected patients who cannot tolerate TMP-SMX, atovaquone and dapsone were similarly effective for the prevention of PCP as compared to dapsone or aerosolized pentamidine [13]. These and the present findings indicate that atovaquone is an alternative prophylactic agent for PCP prophylaxis.

In the present study, $23.9 \%$ of patients among the TMP-SMX discontinued the therapy due to AEs. The discontinuation rate is considered to be much higher as compared to previously reported studies among patients without CTD $[4,14]$. There have been some studies that reported a higher discontinuation rate among CTD patients due to AEs $[7,15]$. In a retrospective study involving Japanese patients with CTDs who started PCP prophylaxis, 43\% (41/96) discontinued TMP-SMX due to AEs [7]. In another study investigated Japanese CTDs patients on high-dose GCs, the discontinuation rate of single-strength TMPSMX was $20.7 \%(12 / 58)$ owing to AEs within the period of 24 weeks [15]. Although the reasons for higher discontinuation rate among certain patient population are not entirely clear, there are several plausible explanations. First, older adults may be at risk of developing AEs from TMP-SMX more frequently due to age-related physiological and pharmacokinetic changes [16]. Second, SLE patients are reported to have higher frequencies of sulfa allergy [17]. Third, reported AEs could be related to flare of CTDs or influence of concomitant drugs for CTDs, such as cyclophosphamide. Half dose of daily single-strength TMP-SMX tablet regimen or dose-escalation regimen may be considered in light of lower frequency of AEs, but their 
efficacies of those PCP prophylaxis regimens have not been proven yet $[15,18]$. In fact, we experienced 2 PCP cases in the TMP-SMX who were administered with less than half dose of daily single-strength TMPSMX. Therefore, alternative prophylactic agents may be preferred in case patients are intolerant of TMPSMX.

Atovaquone is an antiprotozoal drug that is used for the prevention of PCP for patients who are intolerant of TMP-SMX. Among non-HIV patients, PCP prophylaxis with atovaquone is reported to be beneficial for patients following transplantation $[11,12]$. Although atovaquone is a well-tolerated drug, physicians should pay attention to patients' medication adherence because the bioavailability of atovaquone is significantly reduced when administered in the fasting state as compared to be taken after a meal [19]. Lower plasma concentration may lead to inadequate prophylaxis. Previously, 2 cases of prophylaxis failure were reported in transplant recipients who were receiving low dose of atovaquone [20].

The present study had several limitations. First, because it was a single-center study, these results may not be generalizable to a different patient population. Second, the number of PCP cases was relatively small. Third, our study was based on medical record review and some documentation may have been incomplete. Finally, majority of the patients in the atovaquone initially received TMP-SMX for PCP prophylaxis. Although their PCP incident rate may be affected, the impact was probably small over the period of 1 year because all of them stopped TMP-SMX within 4 weeks.

The strengths of this study include the use of data based on relatively large number of CTDs patients receiving high-dose GCs with detailed clinical data including both clinical outcomes and adverse events. We used sophisticated statistical models adjusting for potential confounders using inverse probability of treatment weighting and the Fine-Gray hazard competing risk regression model.

In conclusion, prophylactic effects for PCP in CTDs patients receiving prolonged high-dose GCs were similar between TMP-SMX and atovaquone. Atovaquone could be used for PCP prophylaxis in CTDs patients who are intolerant of TMP-SMX. Further studies should aim to replicate our results in a larger scale.

\section{Declarations}

Ethics approval and consent to participate

This observational study was conducted as per the Declaration of Helsinki. The study was approved by the ethics committee of Kobe University (approval number B190296). In our institute, the institutional review board waived the requirement for patients' informed consent because this study utilized only existing data collected in clinical practice.

Competing interests

All authors have declared no conflicts of interests. 
Author contributions

Conceived and designed the study: SJ and KA. Analyzed the data: SJ and AO. Collection of data: SJ, KA, YN, MY, and JS. SJ prepared the initial draft of the manuscript. All the authors were involved in revising the manuscript critically for content. All authors read and approved the final manuscript.

Data availability

The datasets generated and/or analyzed during the current study are not publicly available. Patients did not provide consent for raw data sharing during the data collection. All aggregated data relevant to the study are included in the article or uploaded as supplementary information.

\section{References}

1. Limper AH, Knox KS, Sarosi GA, Ampel NM, Bennett JE, Catanzaro A, Davies SF, Dismukes WE, Hage CA, Marr KA, Mody CH, Perfect JR, Stevens DA, American Thoracic Society Fungal Working Group. An official American Thoracic Society statement: Treatment of fungal infections in adult pulmonary and critical care patients. Am J Respir Crit Care Med. 2011;183(1):96-128.

2. Park JW, Curtis JR, Moon J, Song YW, Kim S, Lee EB. Prophylactic effect of trimethoprimsulfamethoxazole for pneumocystis pneumonia in patients with rheumatic diseases exposed to prolonged high-dose glucocorticoids. Ann Rheum Dis. 2018;77(5):644-9.

3. Vananuvat P, Suwannalai P, Sungkanuparph S, Limsuwan T, Ngamjanyaporn P, Janwityanujit S. Primary prophylaxis for Pneumocystis jirovecii pneumonia in patients with connective tissue diseases. Semin Arthritis Rheum. 2011;41(3):497-502.

4. Smilack JD. Trimethoprim-sulfamethoxazole. Mayo Clin Proc. 1999;74(7):730-4.

5. Jick H, Derby LE. A large population-based follow-up study of trimethoprim-sulfamethoxazole, trimethoprim, and cephalexin for uncommon serious drug toxicity. Pharmacotherapy. 1995;15(4):428-32.

6. Kimura M, Tanaka S, Ishikawa A, Endo H, Hirohata S, Kondo H. Comparison of trimethoprimsulfamethoxazole and aerosolized pentamidine for primary prophylaxis of Pneumocystis jiroveci pneumonia in immunocompromised patients with connective tissue disease. Rheumatol Int. 2008;28(7):673-6.

7. Kitazawa T, Seo K, Yoshino Y, Asako K, Kikuchi H, Kono H, Ota Y. Efficacies of atovaquone, pentamidine, and trimethoprim/sulfamethoxazole for the prevention of Pneumocystis jirovecii pneumonia in patients with connective tissue diseases. J Infect Chemother. 2019;25(5):351-4.

8. Lertnawapan R, Totemchokchyakarn K, Nantiruj K, Janwityanujit S. Risk factors of Pneumocystis jeroveci pneumonia in patients with systemic lupus erythematosus. Rheumatol Int. 2009;29(5):4916.

9. Sax PE, Komarow L, Finkelman MA, Grant PM, Andersen J, Scully E, Powderly WG, Zolopa AR. AIDS Clinical Trials Group Study A5164 Team: Blood (1->3)-beta-D-glucan as a diagnostic test for HIV- 
related Pneumocystis jirovecii pneumonia. Clin Infect Dis 2011, 53(2):197-202.

10. Warny M, Helby J, Nordestgaard BG, Birgens H, Bojesen SE. Lymphopenia and risk of infection and infection-related death in 98,344 individuals from a prospective Danish population-based study. PLoS Med. 2018;15(11):e1002685.

11. Gabardi S, Millen P, Hurwitz S, Martin S, Roberts K, Chandraker A. Atovaquone versus trimethoprimsulfamethoxazole as Pneumocystis jirovecii pneumonia prophylaxis following renal transplantation. Clin Transplant. 2012;26(3):E184-90.

12. Colby C, McAfee S, Sackstein R, Finkelstein D, Fishman J, Spitzer T. A prospective randomized trial comparing the toxicity and safety of atovaquone with trimethoprim/sulfamethoxazole as Pneumocystis carinii pneumonia prophylaxis following autologous peripheral blood stem cell transplantation. Bone Marrow Transplant. 1999;24(8):897-902.

13. Chan C, Montaner J, Lefebvre EA, Morey G, Dohn M, Mclvor RA, Scott J, Marina R, Caldwell P. Atovaquone suspension compared with aerosolized pentamidine for prevention of Pneumocystis carinii pneumonia in human immunodeficiency virus-infected subjects intolerant of trimethoprim or sulfonamides. J Infect Dis. 1999;180(2):369-76.

14. Jick H, Derby LE. A large population-based follow-up study of trimethoprim-sulfamethoxazole, trimethoprim, and cephalexin for uncommon serious drug toxicity. Pharmacotherapy. 1995;15(4):428-32.

15. Utsunomiya M, Dobashi H, Odani T, Saito K, Yokogawa N, Nagasaka K, Takenaka K, Soejima M, Sugihara T, Hagiyama H, Hirata S, Matsui K, Nonomura Y, Kondo M, Suzuki F, Tomita M, Kihara M, Yokoyama W, Hirano F, Yamazaki H, Sakai R, Nanki T, Koike R, Kohsaka H, Miyasaka N, Harigai M. Optimal regimens of sulfamethoxazole-trimethoprim for chemoprophylaxis of Pneumocystis pneumonia in patients with systemic rheumatic diseases: results from a non-blinded, randomized controlled trial. Arthritis Res Ther. 2017;19(1):7-016.

16. Klotz U. Pharmacokinetics and drug metabolism in the elderly. Drug Metab Rev. 2009;41(2):67-76.

17. Pope J, Jerome D, Fenlon D, Krizova A, Ouimet J. Frequency of adverse drug reactions in patients with systemic lupus erythematosus. J Rheumatol. 2003;30(3):480-4.

18. Takenaka K, Komiya Y, Ota M, Yamazaki H, Nagasaka K. A dose-escalation regimen of trimethoprimsulfamethoxazole is tolerable for prophylaxis against Pneumocystis jiroveci pneumonia in rheumatic diseases. Mod Rheumatol. 2013;23(4):752-8.

19. Freeman CD, Klutman NE, Lamp KC, Dall LH, Strayer AH. Relative bioavailability of atovaquone suspension when administered with an enteral nutrition supplement. Ann Pharmacother. 1998;32(10):1004-7.

20. Rodriguez M, Sifri CD, Fishman JA. Failure of low-dose atovaquone prophylaxis against Pneumocystis jiroveci infection in transplant recipients. Clin Infect Dis. 2004;38(8):e76-8.

\section{Tables}


Table 1. Baseline characteristics of connective tissue diseases patients receiving high-dose glucocorticoids 


\begin{tabular}{|c|c|c|c|}
\hline Characteristic & $\begin{array}{l}\text { TMP-SMX } \\
(n=281)\end{array}$ & $\begin{array}{l}\text { Atovaquone } \\
(n=107)\end{array}$ & P-value \\
\hline Age, mean (SD) & $56(18)$ & $60(18)$ & 0.04 \\
\hline Male sex, n (\%) & $99(35.2)$ & $31(29.0)$ & 0.24 \\
\hline Disease duration, months mean (SD) & $42(80)$ & $49(77)$ & 0.57 \\
\hline \multicolumn{4}{|l|}{ Diagnosis, n (\%) } \\
\hline Systemic lupus erythematosus & $58(20.6)$ & $25(23.4)$ & 0.56 \\
\hline PM/DM & $55(19.6)$ & $23(21.5)$ & 0.67 \\
\hline ANCA associated vasculitis & $53(18.9)$ & $20(18.7)$ & 0.97 \\
\hline Rheumatoid arthritis & $22(7.8)$ & $10(9.3)$ & 0.63 \\
\hline Aortitis & $20(7.1)$ & $4(3.7)$ & 0.03 \\
\hline Behcet's disease & $6(2.1)$ & $2(1.9)$ & 0.86 \\
\hline Adult onset Still's disease, n (\%) & $7(2.5)$ & $4(3.7)$ & 0.52 \\
\hline IgG4-related disease, n (\%) & $21(7.5)$ & $4(3.7)$ & 0.17 \\
\hline Mixed connective tissue disease, $\mathrm{n}(\%)$ & $3(1.1)$ & $4(3.7)$ & 0.08 \\
\hline *Other diseases, n (\%) & $36(12.8)$ & $11(10.3)$ & 0.48 \\
\hline Initial PSL dose, mg/day, mean (SD) & $42.7(15.5)$ & $46.0(13.7)$ & 0.05 \\
\hline \multicolumn{4}{|l|}{ Concomitant treatment, n (\%) } \\
\hline Sulfasalazine & $10(3.6)$ & $3(2.8)$ & 0.71 \\
\hline Steroid pulse therapy & $36(12.8)$ & $29(27.1)$ & $<0.01$ \\
\hline Cyclophosphamide & $40(14.2)$ & $21(19.6)$ & 0.19 \\
\hline Biologics & $29(10.3)$ & $8(7.5)$ & 0.39 \\
\hline 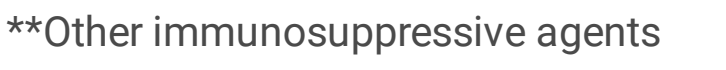 & $101(35.9)$ & $33(30.8)$ & 0.34 \\
\hline \multicolumn{4}{|l|}{ Underlying disease, n (\%) } \\
\hline Chronic lung disease & $112(39.9)$ & $55(51.4)$ & 0.04 \\
\hline Diabetes mellitus & $85(30.2)$ & $26(24.3)$ & 0.25 \\
\hline Lymphocyte count & $78(28.0)$ & $47(43.9)$ & $<0.01$ \\
\hline $800 / \mu \mathrm{l}$ & & & \\
\hline
\end{tabular}

Data are no. (\%) patients or mean (SD) 
Demographic and clinical characteristics as mean for continuous data and as numbers (percentages) for categorical data. T-test or Wilcoxon signed-rank test and the chi-squared test or fisher's exact test were used to compare the clinical characteristics between the 2 groups for continuous variables and categorical variables, respectively.

*Other diseases included cryoglobulinemia, polyarteritis nodosum, polymyalgia rheumatica, relapsing polychondritis, Sjogren's syndrome, and systemic sclerosis.

**Other immunosuppressive agents included azathioprine, methotrexate, mizoribine, and mycophenolate. ANCA, anti-neutrophil cytoplasmic antibody; IgG, immunoglobulin G; PM/DM, polymyositis/dermatomyositis; PSL, prednisolone; TMP-SMX, trimethoprim-sulfamethoxazole

\section{Figures}

Figure 1. Overview of decisions regarding pneumocystis pneumonia prophylaxis

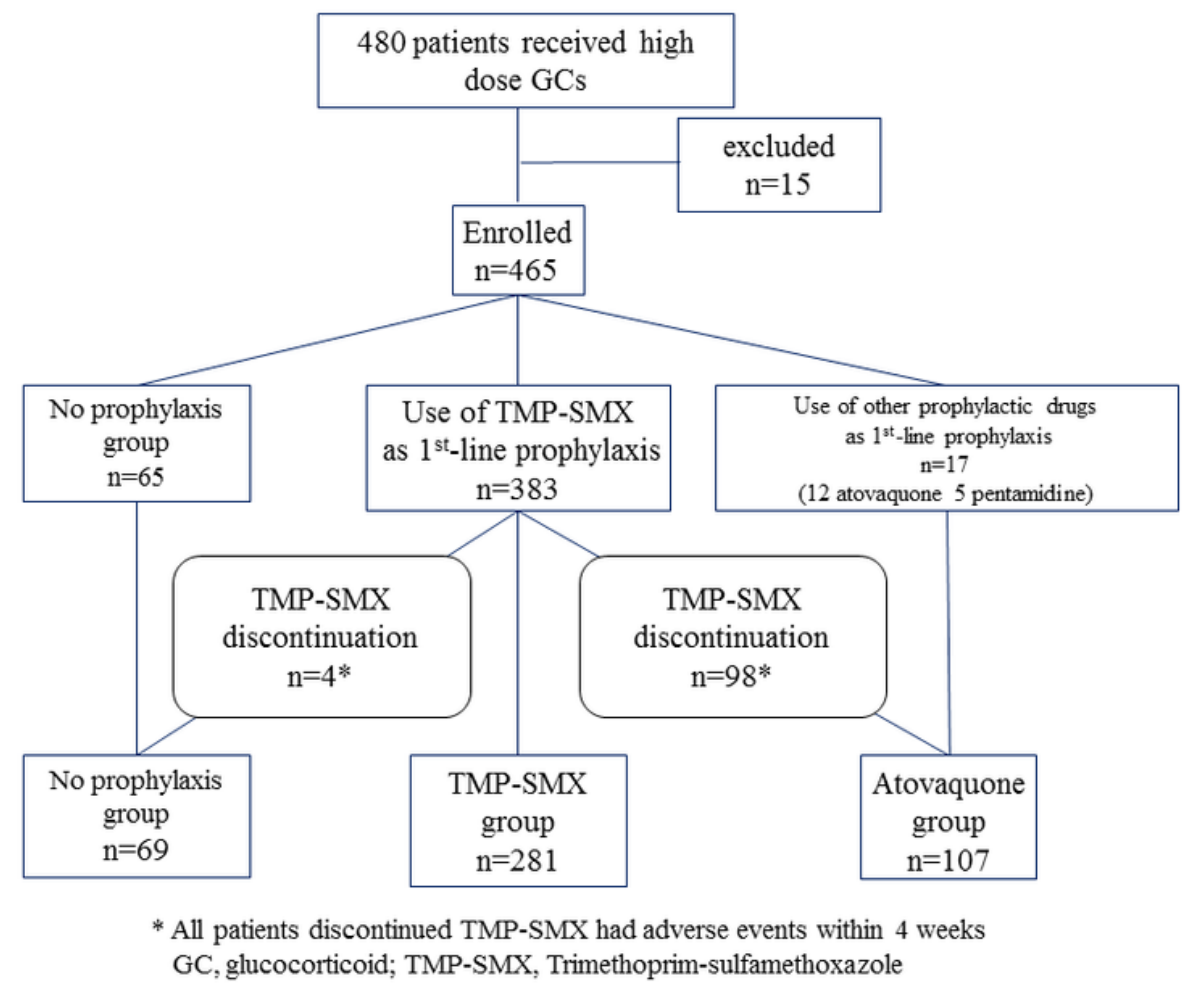

\section{Figure 1}

shows overview of decisions regarding pneumocystis pneumonia prophylaxis. Out of 102 patients who discontinued TMP-SMX, 98 switched to other prophylactic drugs (95 atovaquone and 3 pentamidine), and 4 stopped PCP prevention. Subsequently, patients were categorized as follows; 281 patients to TMPSMX group, and 107 patients to atovaquone group. 
Figure 2. Adjusted pneumocystis pneumonia cumulative incidence of each prophylaxis group.

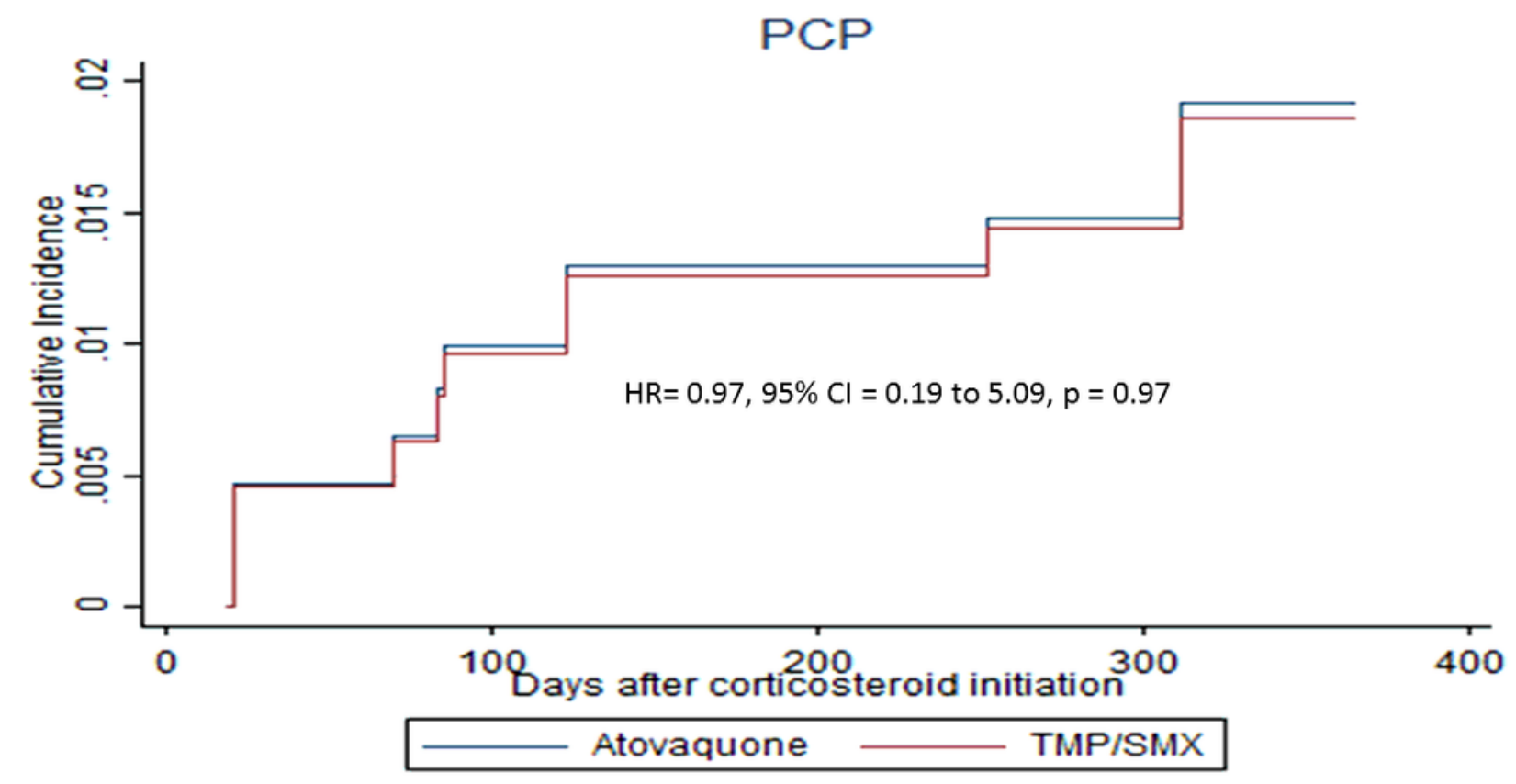

\section{Figure 2}

shows the adjusted cumulative incidence of $\mathrm{PCP}$ was similar between the two group $(\mathrm{HR}=0.97,95 \% \mathrm{Cl}=$ 0.19 to $5.09, p=0.97)$.

\section{Supplementary Files}

This is a list of supplementary files associated with this preprint. Click to download.

- SupplementaryTABLES1.docx 